\title{
Study on the residual stress of bar with straightening by two rolls
}

\author{
Yufeng Wang ${ }^{1}$, Junkai Fan², Cai Liu ${ }^{1}$
}

1. School of Mechanical Engineering, Yanshan University, Qinhuangdao 066004, China,

2. School of Mechanical and Power Engineering, Henan Polytechnic University, Jiaozuo 454000, China

Key words: residual stress; straightening; bar; 42CrMo; finite element method

Abstract: The 42CrMo bar has been widely used for its outstanding mechanical performance. Before bar leaves rolling factory, the straightening process should be used to keep bar's straightness. However, the residual stress is also generated simultaneously, which largely affects the bar in using. In this article, the residual stress of $42 \mathrm{CrMo}$ bar with two roll straightening process was studied by finite element method. The results show that, after the straightening process, the compressive stress and tensile stress were generated on bar's surface and center, respectively. Meanwhile, the bar's neutral layer was migrated. The bar's residual stress was tested with hole-drilling technology. The comparison between the simulation and test results has been proved the validity of the finite element analysis. The results and methods proposed here is the basis of optimizing straightening process.

\section{Introduction}

The medium carbon alloy steel 42CrMo has many good performances, such as high strength and toughness, no obvious temp brittleness. After quenching and tempering treatment, this steel is of high fatigue limit, anti-shock ability, and good impact toughness at the low temperature. For these good performances, this kind of steel has been obtained wide applications in many key components, such as engine cylinder and crankshaft[1].

In the production of $42 \mathrm{CrMo}$ bar, due to the high collecting temperature, the bar is easy to cause bending. Therefore, after rolling, the $42 \mathrm{CrMo}$ bar must be straightened before out of factory. In all kinds of straightening equipment of bar, the two roller straightening machine has the advantages of simple structure and high production efficiency, which has become the mainstream of bar straightening. The 3D model of a two roll straightening machine is shown in Figure 1.

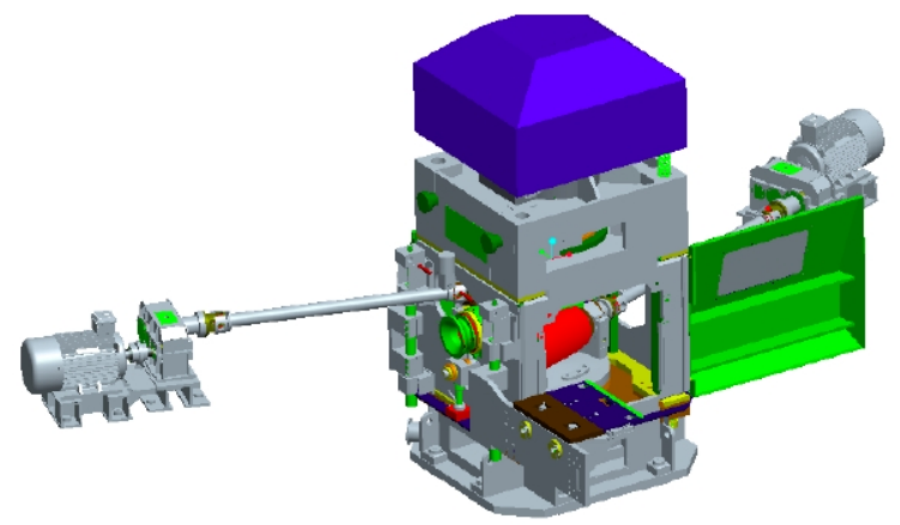

Figure 1. 3D Model of two-roll straightening mechine 
Two roller straightening machine is achieved the straightening ability by its curve gap between the concave and convex curve rollers. When bar is in straightening process, the homogeneous elastic-plastic deformation has been generated with the principle of rotation[2]. The two roll straightening process and the straightening principle is very different from the more inclined roll straightening. Its straightening quality does not depend on the straightening roll number and configuration, but largely depends on the shape of roll. Roll shape must ensure a reverse curvature is produced between the two rolls. The variation of reverse curvature can be divided into three part along the roll direction. Around the bar inlet, the curvature is varying from small to large, then keep a constant value, and gradually decreasing into a small value. The straightening effect of this kind of straightening machine is continuous, and its straightening effect is better than other methods.

The straight degree of the bar is directly related to its residual stress after straightening process. In addition, the material utilization ratio of the $42 \mathrm{CrMo}$ steel bar is largely depends on bar's straight degree. The two roll straightening is a complicated elastic and plastic problem. So far, there is no good way to solve the stress and strain of the bar in analytical solution. In this article, the residual stress of bar after two roll straightening process was studied with finite element method. In addition, the bar's residual stress was tested with the hole-drilling technology. The comparison between the simulation results and test results proves the validity of the finite element model. The research results and methods have important significance for optimizing the straightening process.

\section{FEA model}

The bar two roll straightening equipment can be divided into six parts, which are convex roller, concave roller, front guide sleeve, front guide plate, back guide sleeve and back guide plate. The combining form of these parts is shown in Figure 2.

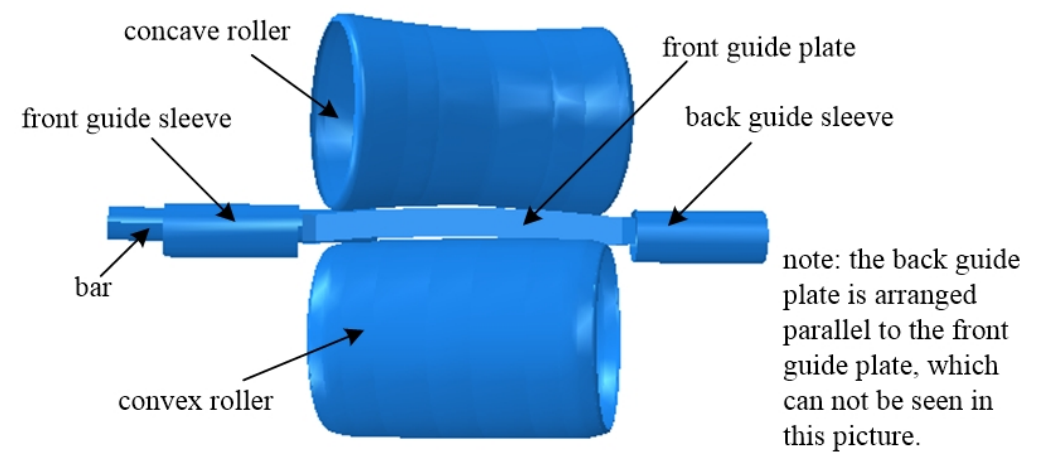

Figure 2. Assembly of two-roll straightening model

In the analysis model, bar's length is $1000 \mathrm{~mm}$, diameter is $120 \mathrm{~mm}$. In addition, the origin deflection of this bar was set as $10 \mathrm{~mm} / \mathrm{m}$. The finite element commercial software Abaqus was used in the simulation. Because of bar's stiffness is very small compared with the six parts in the two roll straightening equipment (as shown in figure 1), in the analysis model, the bar is set as an elastic-plastic body, and the six parts are all set as discrete rigid body. This not only reduces the complexity of the calculation, but also in line with the actual situation. Meanwhile, it is conducive to the convergence of the simulation. The bar is meshed with 30735 C3D8R elements, and the others are meshed with 15420 R3D4 elements. The friction behavior between roll and bar is taken into account in the analysis, and the friction coefficient is set as 0.2. The finite element model after meshing is shown in Figure 3. 


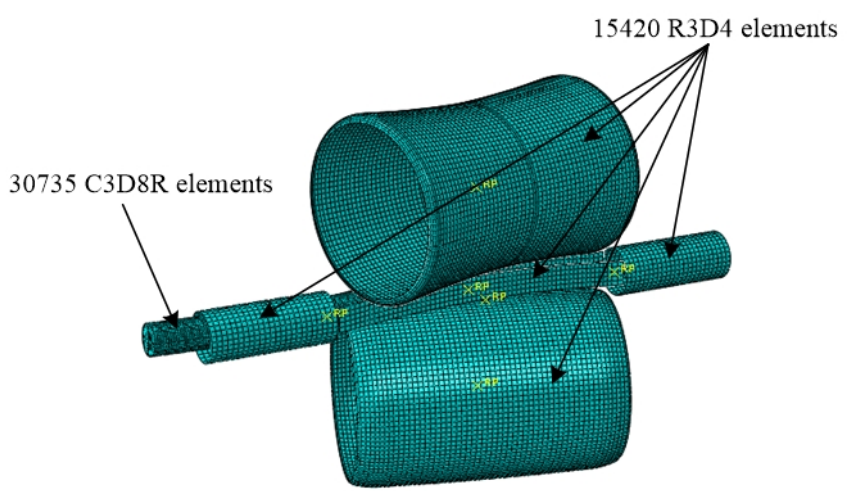

Figure 3. Finite element model

The mechanical property of $42 \mathrm{CrMo}$ bar was tested by tensile testing machine. Deduced from the test results, the bar's Elastic modulus is $210 \mathrm{GPa}$, Poisson ratio is 0.3 and Yield stress is 930MPa. The Von Mises yield criterion was used in the analysis, and the test results of plastic strain versus yield stress were listed in Table.1.

Table 1. Material data of testing

\begin{tabular}{lccccccc}
\hline Plalastic strain & 0 & 0.005 & 0.01 & 0.02 & 0.03 & 0.05 & 0.07 \\
\hline Yield stress (MPa) & 930 & 990 & 1020 & 1060 & 1075 & 1100 & 1120 \\
\hline
\end{tabular}

The bar's initial entry velocity should be matched with the line velocity of rolls. In order to prevent the bar's velocity too fast, its speed is set slightly smaller than the speed of the straightening speed. The relationship between bar's inlet velocity and roll's surface linear velocity can be expressed as:

$$
v=v_{g} \sin \alpha=\frac{\pi D_{g} n_{g}}{60} \sin \alpha
$$

where $v$ is bar's inlet velocity $(\mathrm{m} / \mathrm{s}), v_{g}$ is roll's surface linear velocity $(\mathrm{m} / \mathrm{s}), D_{g}$ is roll's diameter $(\mathrm{m}), n_{g}$ is roll's rotational velocity $(\mathrm{r} / \mathrm{min})$ and $\alpha$ is angle of inclination for straightening roller $\left({ }^{\circ}\right)$. The straightening speed was set as $10 \mathrm{~m} / \mathrm{min}$.

\section{Results and discussion}

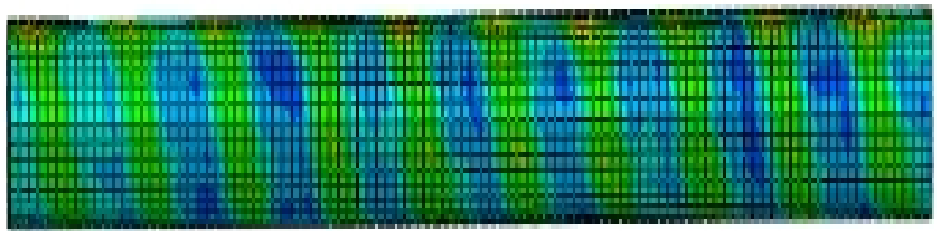

Fig.4 Mises stress contour at stale area of bar after straightening

Around bar's two ends, the stress is largely fluctuated because of the impact phenomenon when bar's inlet and outlet of rollers. For easy to discuss, the results were only extracted from bar's stability straightening part. The distribution of Von Mises stress on bar's surface after straightening process is shown in Figure 4. It can be seen that the bar's surface stress is of the spiral distribution, which is consist with the two rollers straightening process. 


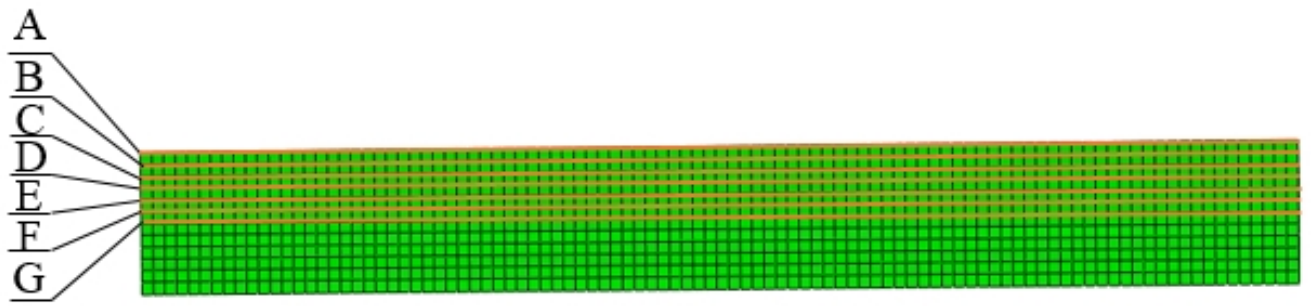

Fig. 5 Paths for radial stress extraction

Based on the character of stress spiral distribution, the results of residual stress can be extracted on bar's center symmetric cross section. The results extraction paths are shown in Figure 5. Form bar's surface to its center, there are seven results extraction paths were allocated.

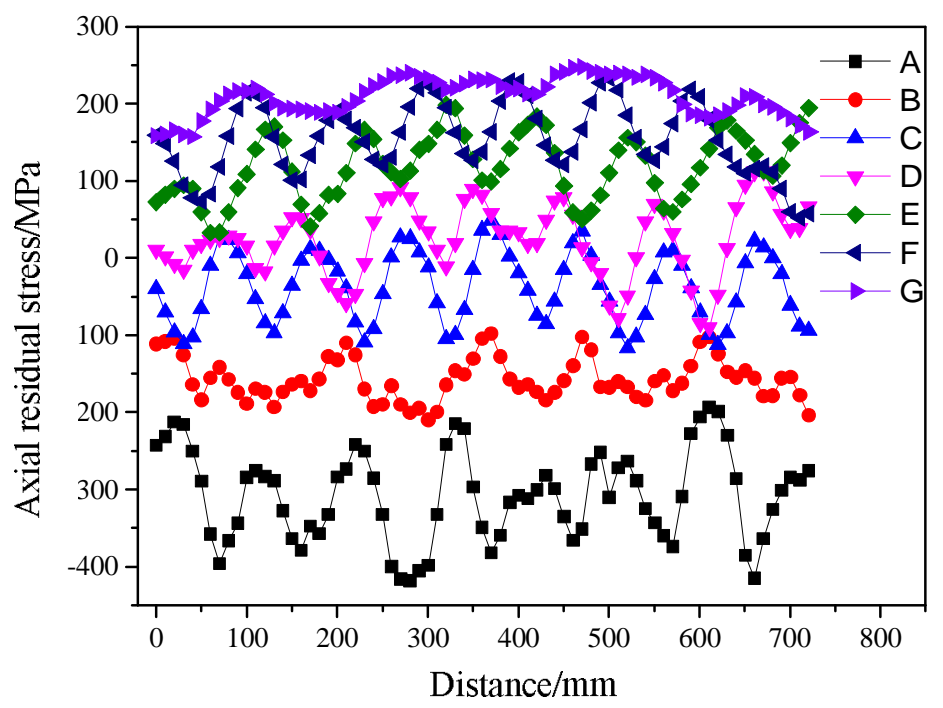

Fig. 6 Axial residual stress distribution along different paths

The axial residual stress along the seven paths are shown in Figure 6. It can be seen that the residual stress is varying from bar's surface to center. On bar's surface, the residual stress in compressive stress, however, the tensile stress is generated around bar's center. In addition, the absolute value of the surface compressive stress is larger than the tensile stress at bar's center. It can be deduced that the bar's geometric middle layer is not the middle layer of the stress. The bar's middle layer has been deviated. This is not taken into account in many analytical analysis models.

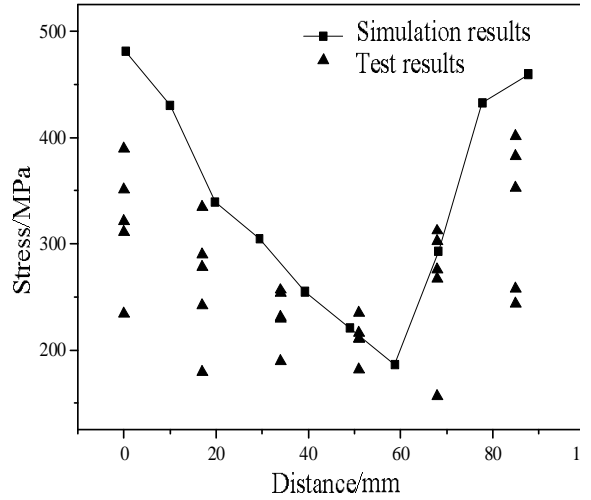

Fig.7 Diagram of residual stress measure using blind-hole method

The bar's residual was tested with hole-drilling technique. The hole-drilling technique can test the residual stress (- , on a two dimensional plane. In the center of the plane, a hole should be 
drilled, and make sure that the depth of the hole is 1.2 times its diameter. After hole has been generated, the residual stress would be released, and an additional strain is emerged which can be tested by strain gauge around the hole. The arrangement of the strain gauges around the hole is shown in Figure 7. The relationship between the gotten strain and residual stress can be expressed as[3-5]:

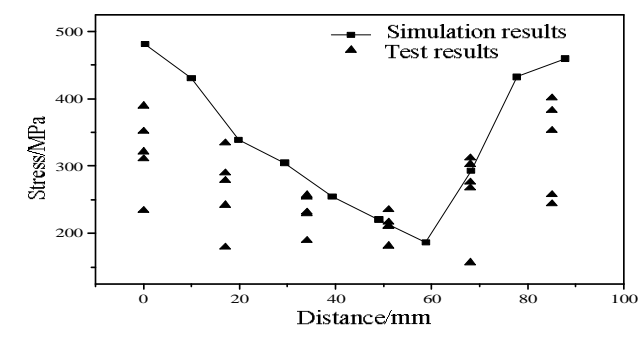

where and are principal stress of the residual stress around this hole, released strains, $A$ and $B$ are the stress release coefficient, is the angle between principal stress and strain gauge.

Based on the principal stress calculated in equation (2), the equivalent stress of residual stress can be deduced as:
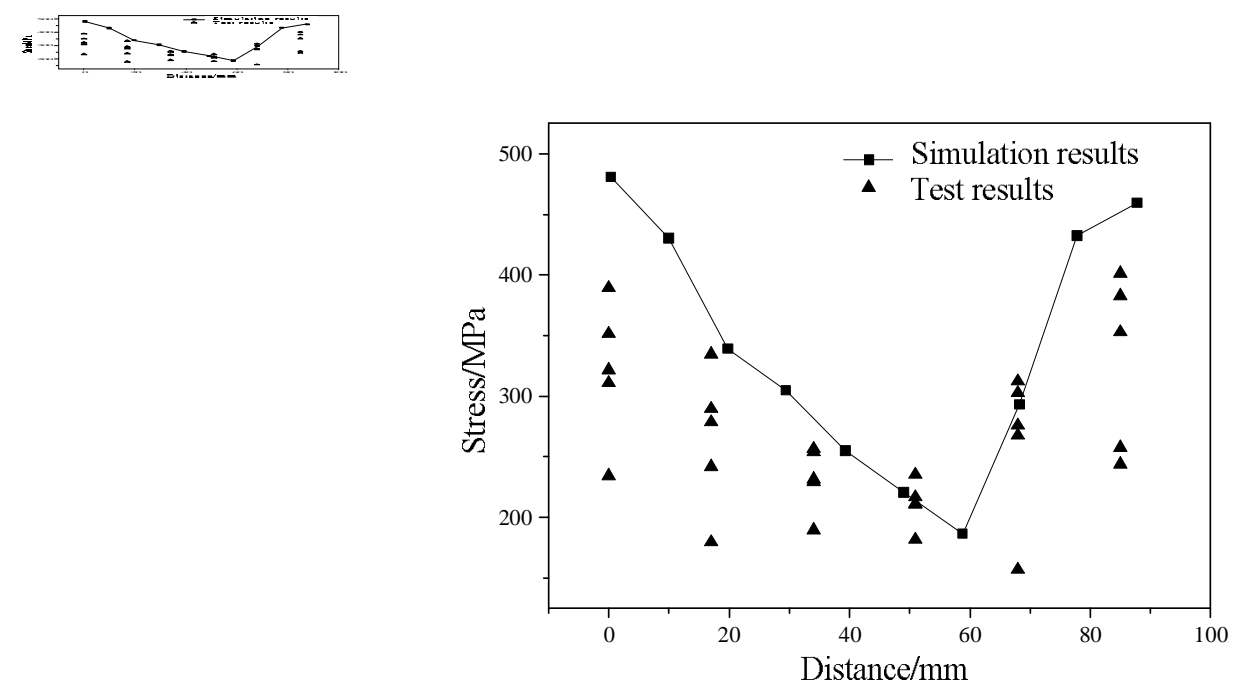

Fig. 8 Comparison of residual stress between test and FEM

The distribution of the test residual stress along bar's length is shown in Figure 8, and these results are compared with the residual stress derived from simulation. It can be seen that the test results are all smaller that the simulation results. However, they have the same varying form. The reason for this can be presumably connected with the fact that the test results are gotten on two dimensional plane, and the simulation results are derived from three dimensional stress field.

\section{Conclusions}

In this article, the finite element model of bar's two roll straightening process has been established. In addition, bar's residual stress is derived from the simulation results and compared with the test results. The results show that the residual stress is varying from bar's surface to center. On bar's surface, the residual stress in compressive stress, however, the tensile stress is generated around bar's center. The bar's geometric middle layer is not the middle layer of the stress, which is not taken into account in many analytical analysis models. The proposed finite element model and simulation results provides the basis for the optimization of straightening process in the future. 


\section{Acknowledgements}

We acknowledge the support by National Natural Science Foundation of China (Grant NO.51405136), and Doctor Foundation of Henan Polytechnic University (Grant NO.B2015-37).

\section{References:}

[1] Y.C. Lin, Ming-Song Chen, Jue Zhong. Prediction of 42CrMo steel flow stress at high temperature and strain rate, Mechanics Research Communications, 2008, 35(3): 142-150.

[2] Dachao Hu, Haomin Chen. The Design and Study of Roller Curvature for the Tube or Bar Straightener, Advanced Materials Research, 2011, 145:394-398.

[3] ASTM Designation: E837-81. Standard test method for determining residual stresses by the hole-drilling stain method. Annual Book of SATM Standard. 1993, (3):01.

[4] J. Malhar. Determination of Initial Stress by Measuring the Deformation around Drilled Hole. Tran. ASME, 1934, 56: 245-254.

[5] W. Soete, R. Vancrombrugge. An industrial Method for the Determination of Residual Stresses, 1950, 8(1): 17-28. 